\title{
EL CAMINO HACIA UNA NUEVA ANTROPOLOGÍA CLÍNICA: AFECTIVIDAD Y CORPORALIDAD EN LA OBRA DE ERWIN STRAUS
}

\author{
The way towards a new clinical anthropology: affectivity and corporality in Erwin Straus's work \\ O Caminho até uma Nova Antropologia Clínica: Afetividade e Corporalidade na Obra de Erwin Straus
}

SEbASTIÁn EdUARDO MEndL

\begin{abstract}
Resumo: A partir de uma leitura hermenêutica da fenomenologia antropológica de Erwin Straus, procura-se elucidar o sentido original da afetividade e a corporalidade, como modalidades essenciais que constituem a relação da existência humana com seu mundo. Primeiro, por meio duma reconsideração histórico-critica de certas proposições da psicologia científica, e de seus fundamentos filosóficos, é preciso retornar ao origem da condição humana, deformada pelas interpretações fundadas nos supostos a priori, para desvenda-la. Precisamente, frente à psicologia naturalista atual e invisibiliza a diferença ontológica entre o homem e as coisas, duma perspectiva strausiana pretende-se propor como objeto primário de estudo da ciência psicológica a subjetividade encarnada, que sente-se a sim mesma quando sente outra coisa, e sua própria ação está continuamente exposta a outras influências, daí sua vulnerabilidade intrínseca. As presentes análises permitem estabelecer um novo marco, tanto para as investigações experimentais futuras quanto para a compreensão da experiência do homem alienado. Por um lado, a antropologia fenomenológica de Straus permite cortar com os três dualismos que sustentam as investigações contemporâneas da corporalidade e a afetividade: sujeito-objeto, receptividade-atividade e interior-exterior. Em segundo lugar, agora, as patologias psico(pato)logias são entendidas como modos diversos de relações do eu-mundo, plenamente significativas, nas quais prevalece a impossibilidade da existência de decidir sobre o curso de sua biografia.
\end{abstract}

Palavras-chave: Psicopatologia; Fenomenología; Afectividad; Cuerpo; Psicología naturalista.

\begin{abstract}
From a hermeneutic reading of the phenomenological anthropology of Erwin Straus, current work seeks to explain the original meaning of affectivity and corporality, as constituting modalities of the relation between human existence and its world. On the first term, through a critical-historical reconsideration of certain propositions of scientific psychology and their philosophical foundations, the aim is to go back to the origin of human condition, deformed by interpretations founded on suppositions a priori, uncovering it. Precisely, opposite to current naturalistic psychology, fades off the ontological difference between man and things, from a perspective as Straus', the incarnated subjectivity, which feels itself when it feels something else, is proposed as the primary object of study of psychological science; its own action is exposed continuously to other influences, therefore, its intrinsic vulnerability. The present analysis allows us to establish a new frame, both for the future experimental investigations and for the comprehension of the experience of the alienated man. Indeed, Straus' phenomenological anthropology allows us to break off the three dualisms that support contemporary investigations of corporality and affectivity: subject-object, receptivity-activity, interior-exterior. Secondly, psychopathologies are understood as different modalities of I-world relations, fully significative in which the impossibility of existence to decide on the course of his biography prevails.
\end{abstract}

Keywords: Psychopathology; Phenomenology; Affectivity; Body; Naturalistic Psychology.

Resumen: Desde una lectura hermenéutica de la antropología fenomenológica de Erwin Straus, se busca dilucidar el sentido originario de la afectividad y la corporalidad, en tanto modalidades esenciales que constituyen la relación de la existencia humana con su mundo. En primer término, mediante una reconsideración histórico-critica de los fundamentos filosóficos de la psicología científica, se busca retornar al origen, para des-encubrir la condición humana deformada por interpretaciones fundadas en supuestos a-priori. Frente a la psicología naturalista actual, que invisibiliza la diferencia ontológica entre el hombre y las cosas, desde una perspectiva strausiana se propone como objeto estudio de la ciencia psicológica la subjetividad encarnada, que se siente a sí misma cuando siente otra cosa, y su acción propia está expuesta sin cesar a otras influencias, por ello su intrínseca vulnerabilidad. Los presentes análisis permiten establecer un nuevo marco, tanto para las futuras investigaciones experimentales como para la comprensión de la experiencia del hombre alienado. Por una parte, la antropología fenomenológica de Straus permite romper con los tres dualismos que sustentan las investigaciones contemporáneas de la corporalidad y la afectividad: sujeto-objeto, receptividad-actividad e interior-exterior. En segundo lugar, ahora, las psico(pato)patologías se comprenden como diversos modos de relaciones yo-mundo, plenamente significativos, en los que priman la imposibilidad de la existencia de decidir sobre el curso de su biografía.

Palabras-claves: : Psicopatología; Fenomenologia; Afetividade; Corpo; Psicologia naturalista. 


\section{La herencia cartesiana de la psicología científica y la restitución del mundo de la vida.}

La obra de Erwin Straus (1891-1975) constituye el intento mismo de repensar una Psicología que, al establecer un dialogo crítico con sus presupuestos filosóficos, pueda apelarse propiamente una "disciplina científica” (Chessick, 1999; Eng, 1976; Straus, 1966).

Precisamente, para el autor, la Psicología y la Psiquiatría poseen un incuestionado suelo de presupuestos metafísicos e interpretaciones antropológicas que determinan las posibles preguntas y sus modos de resolución (Straus, 1969). En este sentido, la concepción a priori del hombre normal que asumen las diferentes escuelas y corrientes, por más fragmentaria, vaga, viciada que sea, predetermina toda concepción posible de los fenómenos patológicos (Straus, 1966/1971; Thinés, 1977/1978).

En la época contemporánea la teconlogización de la existencia ha fraccionado el objeto de estudio propiamente psicológico, acumulando un vasto conjunto de conocimientos biológicos, pero olvidando al ser-humano como totalidad (Rovaletti, 2016; Straus, 1941; 1969). Mas aún, como destaca Landgrebe (1968), las de-formaciones científicas han convertido al mundo en un lugar insólito: "Cuanto más ha penetrado la ciencia en todas las conexiones de nuestro mundo, cuanto más han desaparecido los únicos rincones oscuros del misterio, tanto más parece escapársenos el mundo y tanto más parece devenir extraño e incomprensible” (Landgrebe, 1968/1975, p. 195).

Por ello, en su determinación por comprender la experiencia del hombre alienado, tal como se encuentra en la práctica clínica, Straus se enfrenta repetidamente con la tarea de comprender la norma, y los axiomas de la vida diaria deformados por la interpretación científica (Chessick, 1999; Fischer, 1976; Zaner, 2004).

Ahora bien, en contraste a otros intentos de fundamentación de la ciencia psicológica, la perspectiva fenomenológica adoptada por Straus no parte de supuestos a-priori, y permite un retorno a lo efectivamente experimentado previo a toda deformación de la vida (Husserl, 1913/1962). Se trata de un modo de aproximación que, al neutralizar, dejar fuera de juego, todo conocimiento que no se justifique en una vivencia inmediata, permite mayores niveles de comprensión de las problemáticas estudiadas (Husserl, 1913/1962). En este sentido, Binswanger (1957/2012) comentando el caso Suzanne Urban traduce el lema husserliano “ihacia las cosas mismas!”, cuando insiste que es necesario dejarse conducir y llevar progresivamente por la naturaleza de los fenómenos.

No obstante, a diferencia de sus colegas psiquiatras de orientación fenomenológica que, ante las limitaciones de la psiquiatría naturalista y objetivista, buscan encontrar en la obra de diferentes filósofos el marco ontológico definitivo para la ciencias humanas
(Fischer, 1976), Straus opta por aplicar una autentica epojé filosófica , y, partiendo de la praxis psiquiátrica, del encuentro con el hombre padeciente, erige su propia antropología filosófico-clínica (Straus, 1966/1971; Titelman, 1976; Zaner, 2004).

En primer término, el autor busca recuperar la experiencia originaria de la corporalidad y la afectividad, en tanto modalidades esenciales que constituyen la relación de la existencia humana con su mundo y determinan todos los comportamientos posteriores (Gennart, 1986; Straus, 1935/1963; 1969). Busca así retornar al origen, des-encubriendo la condición humana deformada por interpretaciones fundamentadas en supuestos a-priori mediante una integración de descripción y el examen histórico-crítico de ciertas proposiciones de la psicología científica y de sus fundamentos filosóficos (Straus, 1969).

Para el autor, será el paradigma de Descartes y Galileo el soporte desde el cual se desarrollan las ciencias occidentales en todas sus variantes, entendidas como un intento de determinación cognoscitiva del mundo en-sí leído en términos físicos-matemáticos (Straus, 1969). La tradición metafísica que opone la verdad propia de un ser imperecedero e inmutable -la epistéme- a un mundo experimentado como móvil, cambiante, en continuo fluir, pura opinión subjetiva -la mera doxa- sufre un vuelco fundamental en la geometría galileana (Husserl, 1954/2008). Con la obra de Galileo se alcanza la idealización matemática del mundo fáctico (Husserl, 1954/2008; Landgrebe, 1968/1975). Se trata de un proceso de abstracción, ajeno a una intuición efectiva, que presupone un mundo de carácter físico-matemático subyacente a toda experiencia cotidiana, efectivamente vivida (Husserl, 1954/2008). Posteriormente, “junto con la matematización devenida obvia demasiado rápidamente, se produce como consecuencia una causalidad natural en sí cerrada, en la que todo acontecer está determinado de antemano y unívocamente" (Husserl, 1954/2008, p.103).

De este modo, se logra sustituir el mundo cotidiano, el entorno experimentado realmente, por el reino de las realidades exactas: la objetividad deviene sinónimo de tablas y fórmulas, y lo cuantificable se equipara a lo verdadero (Husserl, 1954/2008). Desde esta perspectiva, se hace abstracción de la existencia personal de los sujetos y todas sus peculiaridades que se extienden a las cosas y asuntos de su vida práctica (Husserl, 1952/1997; 1954/2008). Ahora bien, Descartes partiendo de la matematización de la naturaleza busca fundamentar aquello que escapa al acaecer físico-matemático (Husserl, 1954/2008). Instaura entonces la división entre dos reinos, el del espíritu y el de la materia, colocando así las premisas mismas para la fundación rigurosa de la ciencia psicológica cuyo objeto propio será el cogito (Straus, 1966/1971; Husserl, 1954/2008).

Sin embargo, la Psicología objetivista acabará desfigurando y reduciendo el sistema cartesiano 
(Straus, 1966/1971; Lindeboom, 1978; Zaner, 1988). La res cogitans, la conciencia, es interpretada según el modelo de los cuerpos de la física y en un paso ulterior, encarnada en el sistema nervioso, siendo este un mero receptor pasivo de estímulos (Straus, 1966/1971). La apropiación naturalista del cogito cartesiano permite así que toda sentencia del campo psicológico sea plausible de ser formulada en lenguaje físico a fin de lograr proposiciones observacionales susceptibles de cuantificación, verificación y análisis, es decir "un lenguaje bien hecho exento de ambigüedad” (Ramos Gorostiza y González Calvo, 1995, p. 282).

Para la psicología objetivista, la supremacía del cogito sobre el cuerpo por medio de la voluntad deviene en una mera ilusión, ahora las sensaciones, las necesidades corporales y los impulsos se erigen como soberanos absolutos e independientes (Straus, 1935/1963), "el movimiento voluntario fue desenmascarado como el movimiento de una marioneta. Todo comportamiento significativo de un organismo para con su mundo fue anulado" (Straus, 1969, p. 40). La existencia es devaluada a mecanismos explicables por causas controlables. En efecto,

"los movimientos dotados de finalidad no pueden originarse en un sistema nervioso, pues esto implicaría que las nuevas conexiones sinápticas se hubieran realizado por elección anticipando una adaptación significativa a la organización del medio” (Straus, 1966/1971, p. 219).

La acción teleológica se reduce a la conexión e integración de los reflejos, la reacción motora sigue necesariamente al estímulo en un intervalo de tiempo cuantificable (Straus, 1966/1971). Se deforma así la experiencia vivida de un ser activo y teleológico, en pro de una visión determinista del sujeto (Straus, 1966/1971; 1967a). La psicología actual, atada a la tradición de Galileo y Descartes (Straus, 1969; 1936/2015), invisibiliza la diferencia ontológica entre el hombre y las cosas inanimadas.

Por otra parte, la res cogitans, la conciencia, encarnada en el sistema nervioso, es atribuida al organismo individual, pero concebida como una vida interior separada del mundo, confinada a sí misma (Straus, 1969). En efecto, la ciega aceptación del sistema cartesiano no solo redujo al cogito a una mera cosa determinada causalmente, indistinta de los objetos de la naturaleza, también condenó a la psicología objetivista a investigar un sujeto mítico, aislado en soledad intrapsíquica, generando así un conjunto de aporías producto de un punto de partida inadmisible (Straus, 1969).

Precisamente, al negar la existencia del mundo, y por lo tanto la propia existencia corporal, la duda cartesiana encuentra su certeza primaria en un cogito privado de mundo, un hombre que contempla los sucesos como radicalmente exteriores (Straus, 1935/1963). Este sujeto mítico que no pertenece al mundo es impasible, no experimenta transformaci- ón alguna, nada puede afectarle, ya que todo lo que acontece se encuentra en el campo de la res-extensa (Straus, 1935/1963; Barbaras, 2004).

En el paradigma cartesiano no hay lugar para una concepción de la sensibilidad en su especificidad, ni hay modo de reconocer la relación originaria de una subjetividad encarnada con su mundo (Straus, 1935/1963). Por ello, la sensibilidad es concebida sólo como una modalidad degradada del conocer, característica esencial del cogito. Cuando Descartes se pregunta por el sentir, lo hace sólo por la función que éste cumple en la realización de la conciencia de objeto, es decir, la admisión puramente receptiva de afecciones (Straus, 1935/1963). Barbaras (2004) muestra la imposibilidad del paradigma cartesiano de dar cuenta de la relación directa de una subjetividad encarnada con su mundo:

"Con el sistema cartesiano, la unidad de la experiencia sensorial es fraccionada en la dualidad de un movimiento objetivo, extraño al cogito, y una experiencia interna, perteneciente a la conciencia, pero sin embargo oscurecida o confundida por virtud de su relación, que sólo puede ser de tipo causal, con el mundo de la res extensa" (Barbaras, 2004, p.217)

Esta conceptualización cartesiana de la experiencia sensorial conduce al concepto de las "sensaciones" de la Psicología naturalista (Straus, 1935/1963). Si la sensibilidad no es concebida como un modo de relación directa con el mundo, se reduce a una colección de contenidos discretos que afectan a la conciencia: son los datos sensoriales concebibles de una manera físico-objetiva y cuyo punto de partida es la materia (Landgrebe, 1957/1969).

La realidad del mundo exterior ya no es puesta en duda, pero es considerada lejana a cualquier contacto directo. Los estímulos con los que trabaja la fisiología del cerebro no son visibles, audibles ni tangibles, sino que actúan exclusivamente sobre un sistema nervioso, siendo acontecimientos estrictamente privados (Straus, 1935/1963; 1965b). En este sentido, frente a la falta de consideración filosófica de sus colegas, Straus irónicamente pregunta “¿cómo puede comprenderse que un 'aparato mental' ubicado en una caja intracraneal pueda alguna vez alcanzar el así llamado 'mundo exterior' y establecer una comunicación con otros cerebros?” (Straus, 1975, p.141).

En efecto, al reducir los órganos sensoriales a receptores pasivos de excitaciones provenientes de la materia, se introduce el objeto de la percepción en el sistema nervioso, volviendo incomprensible el entendimiento intersubjetivo sobre entes comunes al discurso de los interlocutores (Straus, 1958/1967b). Se reemplaza así la experiencia vivida de la percepción por una abstracción metódica que nadie jamás ha experimentado en carne y hueso (Straus, 1975).

Esta búsqueda generalizada de la base neurofisiológica de la mente que condiciona toda investigación de la corporalidad y la afectividad se funda en un juego metafísico implícito en el que se establece una distinci- 
ón entre dos niveles ontológicos: el del sistema nervioso y su funcionamiento, al que se le adjudica plena realidad. Mientras tanto, la experiencia vivida se interpreta como una especie de fantasmagoría, una superficie engañosa relacionada de algún modo con los verdaderos acontecimientos intracraneales (Straus, 1965b; 1975).

Precisamente, la suposición de un sujeto extramundano, la caracterización del sentir como forma inferior del conocimiento y su determinación como el producto de procesos fisiológicos resultados de la acción del mundo sobre la conciencia, así como la negación de todo movimiento teleológico, son cuatro aspectos de una misma postura metafísica (Straus, 1935/1963; Barbaras, 2004). La psicología objetivista pese a reclamar datos confirmados en la experiencia, reemplaza las "cosas mismas" y la experiencia vivida por constructos metafísicos y metodológicos (Straus, 1935/1963). Los comportamientos y la afectividad no son considerados como modalidades de la existencia, como modos de apertura al mundo, sino restringidos a mecanismos objetivos, y finalmente interpretados como el efecto secundario del sistema nervioso (Landgrebe, 1963/1968).

En consecuencia, la psicología actual acaba desenvolviéndose tanto en la investigación como en la práctica con conceptos operativos y descripciones cuantitativas de los fenómenos (Khun, 1996). Números, estadísticas, tablas, representaciones esquemáticas, dominan en la actualidad el panorama académico. Pero esta Psicología científica exacta, que parte del hombre objetivado, mera sumatoria de mecanismos intracraneales, no puede conceder a la locura y al sufrimiento una significación humana (Khun, 1996). Ante esta situación Binswanger (1995/2006) exige,

En lugar de avanzar desde el cuerpo [Körper], sus 'órganos' y las funciones de éstos (...) y a partir de éstos, a su vez, tratar de abrirse paso a la esfera de la persona, lo que únicamente puede conducir siempre al establecimiento de un psicograma propiamente sin sentido (...), el objetivo primero y más próximo de la psiquiatría es la investigación de la persona y a partir de aquí (...) ordenar (...), en una jerarquía plena de sentido, los restantes métodos de investigación más o menos indispensables. Con otras palabras, en lugar de querer penetrar en el problema de la subjetividad a partir de la objetividad, lo que solamente es posible por una especie de reconstrucción o retraducción, resulta ahora la exigencia, opuesta y mucho más fácil de cumplir, de comprender la objetividad a partir de la subjetividad (p. 436).

\section{La situación primaria del hombre: en- tre la autonomía y la vulnerabilidad.}

Frente al "cuerpo objeto" [Körper] estudiado por la fisiología, plausible de ser descompuesto en partes independientes, y que ocupa un punto en el espacio euclidiano -espacio vacío, tridimensional, homogéneo y a-histórico (Husserl, 1952/1997), Straus (1935/1963) apunta a la elucidación del cuerpo vivido [Leib]. A diferencia del Körper que constituye una idealidad, una substracción teorético-lógica, algo por principio no perceptible en su ser propio, el cuerpo-sujeto es experimentado inmediatamente en el curso de los movimientos y las percepciones (Husserl, 1952/1997; Landgrebe, 1968/1975).

Desde la perspectiva strausiana (1952), el cuerpo constituye una adquisición variable. El infante reclama a la tierra el cuerpo como propio en cuanto éste le obedece, y está a su disposición. Mundo y Self se despliegan simultáneamente (Moss, 1981; Straus, 1969). El sujeto que conquista su cuerpo, primera soberanía del hombre, emerge en dicha reclamación como perteneciendo corporalmente en contrariedad al mundo: unión y separación, se originan simultáneamente y determinan la situación originaria [Ur-situation] del individuo (Straus, 1969). El significado deíctico de mi cuerpo, de mis experiencias... conforma una reivindicación frente al mundo, la cual establece que aquellas parcelas que también pertenecen a la tierra están bajo el dominio del sujeto (Straus, 1952).

De este modo, la corporalidad posee una ambigüedad ontológica, pertenece simultáneamente al hombre en tanto subjetividad encarnada y a la tierra que lo contiene. En la enfermedad, en el dolor o en el cansancio, el cuerpo se devela exclusivamente como parcela del mundo, objeto librado de los caprichos humanos. En esos estados, la potencia del mundo arremete contra la apropiación del cuerpo, poniendo en desequilibrio el dominio y la soberanía alguna vez mantenida por el individuo (Straus, 1969). Más aún, la consideración de casos extremos, como la despersonalización o la desintegración del esquema corporal y los fenómenos de influencia de ciertas esquizofrenias (Galimberti, 1987), muestran que incluso el cuerpo propio, los recuerdos y los pensamientos constituyen una adquisición inestable que puede caer bajo la soberanía del mundo (Straus; 1958/1967b).

Vivir consiste en la progresiva conquista de las capacidades corporales y del ambiente; existir es mantener un frágil equilibro en contraposición a la tierra y a los otros, una conquista continuamente amenazada a derrumbarse frente a la opresión de las circunstancias (Straus, 1969). Ahora bien, previo a todo juicio cognitivo, la situación originaria [Ur-situation] del hombre se establece en una certeza vivida desde la propia corporalidad (Straus, 1969). Desde el primer movimiento de la existencia, las mónadas experimentan la singularidad de la condición humana, ese contacto nunca acabado con un mundo que las precede, del cual son dependientes y del cual conforman una parte (Straus, 1952; 1965a). En efecto, con el desarrollo gradual de la motilidad, el infante descubre la distancia entre las intenciones y su cumplimiento, y el mundo se devela como fuerza oposi- 
tora (Landgrebe, 1977/2017). Levantando su cabeza, gateando, parándose y caminando, el hombre asegura su o-posición frente al mundo, experimenta en la pesadez de la gravedad la fuerza constante e ineludible que debe vencer (Straus, 1952; 1965a).

"El hombre y el animal (...) se erigen contra la gravedad por medio de su propia fuerza; la postura erguida del hombre [así como todo movimiento] (...) requiere el constante esfuerzo renovado (...) la gravedad ciertamente es superada en una manera particular, pero ni el hombre ni el animal es capaz de superarla por completo" (Straus, 1969, p. 34)

En este sentido, Straus $(1952 ; 1965 a)$ destaca que el logro de la postura erguida en el hombre tiene una significación existencial. Al levantarse de la tierra, la existencia funda el "aquí primordial”, que es simultáneamente un pertenecer y mantenerse en o-posición, situación del hombre expresada en el lenguaje mediante la palabra "yo" y la negación (Straus, 1969). El infante la adquiere en un proceso que atraviesa múltiples etapas y fracasos, y posteriormente como adulto debe mantener una firme resistencia y luchar ante la tentación de dejarse caer (Straus, 1966/1971): la o-posición frente al mundo establecida en la postura erguida ha de conquistarse permanentemente, siendo las condiciones fisiológicas requisito necesario, pero no suficiente (Straus, 1966/1971).

Precisamente, desde la perspectiva strausiana, todo movimiento humano es irreducible a la lectura de la física clásica, a la mera sucesión de posiciones en el espacio. Querer moverse sólo le es posible a un sujeto que, al estar implicado en el devenir de su existencia, apunta a ser diferente y se experimenta en su historicidad, en perpetuo estado de llegar-a-ser (Straus, 1935/1963).

Ya sea el movimiento exploratorio en la oscuridad o en la huida frente al dolor, siempre se trata de un movimiento orientado hacia un objetivo temático o hacia una situación diferente a la actual (Straus, 1966/1971). Incluso en el simple reflejo, se trasciende la situación inmediata hacia una meta esperada. La acción no puede reducirse a la fisiología porque en todo acto se abandona la condición presente a favor de un escenario futuro (Straus, 1966/1971; 1936/2015), incluso el gesto más elemental condensa una situación biográfica y social (Straus, 1966/1971).

Por ello, el hombre no habita el espacio como las cosas del universo, sino que articula un espacio vivido como distancia y proximidad entre cosas y personas, como horizonte de proyectividad y campo de acción: el espacio asume una connotación existencial en la medida que el hombre se encuentra dirigido hacia el mundo, anhelando aquello que no posee, deseando llegar a ser aquello que no es (Straus, 1935/1963). Las metas por alcanzar, las posibilidades por consumar, pero también la pesadumbre de la gravedad y las inhibiciones de la situación social, habitan la potencialidad del presente: moverse a sí mismo implica la certeza inmediatamente vivida de poder modificar el estado actual del mundo (Straus, 1935/1963; 1966).

El movimiento teleológico o vivido [veçu], introduce lo posible en el seno de lo real y transforma al mundo en un entramado de opciones (Straus, 1966/1971). El espacio vivido -no el de la geometría- se mide inmediatamente frente a las tareas que el hombre se propone y las posibilidades de las que dispone (Straus, 1966/1971). En cada situación surge lo lejano y lo cercano a partir de que el espacio se deja recorrer por la corriente del deseo o la renuncia, donde está en juego la libertad que decide explorarlo o dejarlo como definitiva separación entre el hombre y la cosa, entre el hombre y los otros (Straus, 1935/1963). Por su parte, el esquema corporal, lejos de constituir una imagen mental del cuerpo, se vive como el conjunto de posibilidades, de direcciones y demarcaciones que se descubren en el contacto con el mundo (Merleau-Ponty, 1945/1993).

Si bien la libertad primaria del hombre, su o-posición al mundo, se funda en la corporalidad, es necesario añadir que desde el momento en que el sujeto es nombrado por sus progenitores, debe asumir una postura frente a la historia, frente a las perspectivas del mundo y el rol proporcionado por los alter-egos (Landgrebe, 1997/2017). En este sentido Straus destaca que la expresión inglesa "to be upright" (estar erguido), así como los vocablos latinos derivados de "rectitud", denotan una significación moral, dado que habla de un sostener las propias convicciones y actuar de acuerdo a ellas ante las presiones de las circunstancias (Straus, 1952; 1965a). El hombre posee la libertad para optar continuamente por fundirse en el mundo, renunciar a su o-posición y aceptar como dogma las perspectivas de la masa; o puede aceptar su radical responsabilidad, mantenerse firme ante las presiones del entorno (Straus, 1969).

\section{El esplendor de la vivencia sensorial: la experiencia gnóstica y la experiencia pática.}

La ciencia físico-natural ha reducido el cosmos a la mera totalidad de entes determinables cuantitativamente y desconectados de su significación histórica para un sujeto viviente, dejando a este vacío de sentido y de afectos (Husserl, 1954/2008; Waldenfels, 2008). Por ello, todo aquello que no se enuncia en el saber como cualitativamente neutro y objetivo, o que no puede servir a ciertos fines en la esfera práctica, es relegado al mero dominio de los sentimientos, estados inmanentes, experiencias privadas del sujeto que traducen los efectos del mundo sobre el cuerpo (Landgrebe, 1957/1969).

En efecto, la teoría contemporánea de las sensaciones resultado de la apropiación naturalista del 
cogito cartesiano devaluado a mero receptor pasivo de estímulos, desdobla la experiencia afectiva en un momento objetivo -la acción del mundo sobre el sistema nervioso- y un momento subjetivo, el estado de ánimo producto de la aprehensión cognoscitiva de lo acontecido (Landgrebe, 1968/1975).

Para Straus (1967b), será la estesiología la encargada de presentar la experiencia sensorial libre de los prejuicios tradicionales. Desde esta perspectiva, el sujeto que percibe no es una conciencia pura ni un aparato mental, sino una subjetividad encarnada afectada corporalmente en el contexto de una situación biográfica. Los sentidos lanzan al hombre más allá de sí mismo, hacia el mundo, sin incorporar ni producir una imagen mental de él (Straus, 1965b). De este modo, la concepción strausiana quiebra el dualismo interioridad-exterioridad que domina a la psicología naturalista.

No existe un primer movimiento objetivo que conecte al hombre con los entes y luego una posterior reacción subjetiva (Straus, 1935/1963). El sujeto se abre al mundo por medio de los sentidos, experiencia que antecede a todo juicio cognitivo y cuya primordialidad reside en que el hombre se encuentra atrapado, e irremediablemente afectado por lo que sucede (Straus, 1967b). Precisamente, la realidad no se obtiene en la valoración formal del acontecimiento, sino que se constituye en la certeza vivida de que "algo me ocurre en el mundo" (Straus, 1967b):

Experimentamos la realidad en una relación personal, no está separada de nosotros; en tanto criatura viviente, formo parte de ella; su dramática concreción me afecta; estoy asido y atrapado (...) [el hombre] no es una mente que emita juicios, no es una conciencia atenta a un mundo exterior, sino un ser humano que experimenta el mundo con su propio cuerpo real. La experiencia real es pre-lógica; no es medida (Straus, 1966/1971, p. 125).

Cada modalidad sensorial abre un aspecto del mundo. La unificación de elementos divergentes -el olor y el gusto, los rasgos táctiles y su sabor- es posible porque el hombre, como único e idéntico en la diversidad de sus sentidos, se orienta hacia lo otro, que es también único e idéntico en la diversidad de sus aspectos y no meras representaciones intra-craneales (Straus, 1967b).

Por otra parte, las zonas sensoriales no sólo son distintas respecto a sus objetivos, sino también en la forma en que articulan al hombre con el mundo. La pluralidad de las modalidades que muestra el entorno, con sus aspectos y perspectivas cambiantes, se correlaciona con los distintos modos en los cuales el hombre ve afectado su ser (Straus, 1966/1971). Así, la actividad de la visión en el abrir y cerrar los ojos permite al hombre comprenderse en sus posibilidades de operar en el mundo logrando que se manifiesten sensiblemente las cosas. Por el contrario, la imposición del sonido, la pasividad frente a los estruendos, develan el irremediable confinamiento del sujeto al mundo, un encierro del que nada lo libera.

Ahora bien, frente a esa tradición de la Psicología y la Filosofía que distingue entre contenido inmanente y objetos trascendentes, Straus (1935/1963; 1966) plantea la distinción entre la experiencia pática y la experiencia gnóstica como dos modalidades de expresión de la relación yo-mundo. Mientras en la primera se manifiesta el carácter fisiognómico del entorno, cuya receptividad predispone a la tonalidad afectiva y al comportamiento del hombre, en la segunda la sensibilidad proporciona un mero valor representativo de objetos concretos (Straus, 1966). En palabras de Straus.

Por momento pático, nos referimos a la comunicación inmediata que establecemos em las cosas sobre la base de sus modos cambiantes de dársenos sensorialmente. Por lo tanto, no relacionamos la dimensión pática (...) em las propiedades fijas y cambiantes de los objetos y esto significa no em objetos capaces de atraernos, asustarnos y oprimirnos a causa de sus propiedades (...) Lo pático es em rasgo característico de la experiencia primordial; por ello es tan difícil de comprender em términos conceptuales, pues corresponde a la comunicación pre-conceptual que mantenemos em las apariencias, inmediatamente presentes y sensorialmente vividas (...) El momento gnósico simplemente desarrolla el qué de lo dado em su carácter de objeto; el momento pático desarrolla el cómo de su ser em cuanto dado (Straus, 1966/1971, p. 27-28).

Desde la perspectiva strausiana, el hombre posee primariamente em mundo por medio de la experiencia pática, relación originaria entre em subjetividad encarnada y la tierra que precede a la aproximación a entes particulares (Straus, 1935/1963). Em la luminosidad del ambiente, em el acaecer de los sonidos, em el juego de las formas... se impone la coloración afectiva del entorno, es allí que el mundo se presenta em su totalidad, ya sea como aterrador o confortable, tedioso o vívido... (Straus, 1935/1963). Dicha coloración afectiva del mundo posee em función omniabarcante; acoge em sí todo tipo de experiencia, todo hacer y todo querer (Straus, 1948). Em palabras de Straus: "Los caracteres fisiognómicos no tienen localización precisa ni em delimitación definida; está indeterminación espacial es sentida como em 'em todas partes', y la falta de delimitación como em alcance más allá de toda distancia" (Straus, 1948, p. 37).

La experiencia pática constituye la modalidad de estar vivo [lebendiges Sein] que es condición de posibilidad de aprehender toda trascendencia 
(Straus, 1935/1963); la certeza sensorial de em mundo exterior no puede ser derivada de percepciones individuales, sino que se establece em la relación em la totalidad dada em el sentir (Straus, 1935/1963). El momento gnóstico de la experiencia se erige posteriormente como diferenciaciones y delimitaciones de la totalidad del mundo dado por la experiencia pática.

De este modo, aquellos caracteres de lo atrayente, repelente, terrible, etc. -reducidos demasiado rápidamente por la Psicología actual a cualidades de expresión, fundadas em cualidades sensibles elementales y em las efectuaciones aprehensivas y cognoscitivas que se estructuran sobre éstas- proporcionan la primera apertura al mundo (Landgrebe, 1968).

Em este sentido, Zirión (2009), destaca que em el entorno, em las múltiples relaciones que se establecen em él, em los demás, em sí mismo, em las actividades del hombre, aficiones, esperanzas, expectativas, temores, sueños, recuerdos, em todo lo que hace a la trama de la vida, se presentan constantemente cambios innumerables. Sin embargo, al hombre no le es necesario recorrerlos uno a uno para apreciar que su singular combinación conforma, a cada instante, em particular colorido de la vida (Zirión, 2009).

El colorido de la vida se distingue del estado de ánimo (Zirión, 2009). El primero no designa em estado interno del hombre, sino cómo el mundo se colorea conforme a la manera como es vivido por la existencia (Zirión, 2009). Por ello, mientras que el estado de ánimo puede vivirse más de em vez, el colorido constituye el resultado de em entramado afectivo de em complejidad incalculable, de em relación em el mundo única e irrepetible (Zirión, 2009). Precisamente, las características fisiognómicas del mundo no se emancipan de la situación biográfica de em subjetividad encarnada (Straus, 1935/1963). El hombre se ve enredado em historias, em la intersección de múltiples tramas inconclusas que exigen em resolución (MacIntyre, 1981); por ello, el mundo y los entes particulares se presentan, a cada momento, de em modo absolutamente único según el devenir de cada biografía humana. Así, em extensa caminata por la montaña adquiere significación diferente para el turista y el nativo, incluso para el viajante cuyo vuelo peligra y para aquel que recorre los senderos em templanza.

No obstante, la fisiognomía del entorno no se reduce a meras impresiones subjetivas. El hombre experimenta la iniciativa de las cosas, se siente afectado em su existencia por la actividad motriz y afectiva que ellas ejercen sobre su persona (Straus, 1967b). El sentir strausiano designa em estrato que escapa o es previo a la distinción entre sujeto y objeto (Gennart, 1986; Maldiney, 1973). Em consecuencia, el paisaje del mundo es captado em mayor lucidez por metáforas, analogías, lenguajes indirectos que expresen em vivencia singular, irrepetible, y no se encuentren restringidos por el dualismo sujeto-objeto de la Psicología objetivista.

Por una parte, el momento pático de la experiencia también implica quebrar el dualismo recepti- vidad-actividad. Dado que el sentir constituye simultáneamente em sentirse a sí-mismo y em apertura a su situación, no se lo caracteriza de modo suficiente si se lo describe sólo como pura recepción pasiva de estímulos (Landgrebe, 1968/1975). Lo aberrante y lo confortante suponen em ser susceptible de acercarse o alejarse, em libertad para comportarse de modo activo frente al entorno: la experiencia pática sólo le es posible a seres que se encuentra em o-posición al mundo (Straus, 1935/1963; Barbaras, 2004). De este modo, la estesiología abre el campo de estudio em la interrumpida relación entre movimiento teleológico y sentir:

La experiencia sensorial no es ni una mera recepción de data hylética ni un puro acto de espontaneidad. Tocando algo, simultáneamente tocamos nuestro propio ser. Para ver las maravillas del mundo debemos viajar, ir hacia donde ellas están; se nos presentan; cuando nos exponemos a ellas, cuando nosotros -como dice el lenguaje cotidiano- le echamos el ojo. Las vemos a la distancia, pero no las ubicamos en un mundo exterior (...) En la experiencia sensorial somos conscientes de las cosas y de nosotros mismos en nuestra existencia corporal. Ésta relación no tiene contraparte en el reino de las cosas inanimadas (Straus, 1965b, p.198).

La distancia, el no poder moverse, la euforia y el estado de sitio pertenecen al contenido original y persistente de la apertura al mundo en el sentir pático. Las expresiones del lenguaje cotidiano "paralizado del susto", "saltando de alegría”, entre otras, develan que la separación del sentir y la corporalidad vivida constituye una abstracción metódica fundamentada en la suposición de la interioridad extra-mundana del cogito cartesiano. El hombre se comprende a sí mismo y su situación

En síntesis, la afectividad y la motilidad se implican mutuamente, y son constitutivas de la existencia humana. Su inter-juego funda la situación primaria [Ur-situation] del hombre: existir en contrariedad a un mundo que lo precede. Por ello, la pregunta por los elementos últimos, es decir, por aquellos aspectos que constituyen la estructura de la conciencia y en los que se fundan todos los restantes, no debe buscarse más en las sensaciones y su posterior aprehensión cognoscitiva, sino en la interacción entre el sentir y el movimiento teleológico.

\section{La psicología strausiana: una ciencia fundamentada ontológicamente en el ser del hombre.}

La Psiquiatría y la Psicología paulatinamente se han ido vaciando de sus cimientos para volcarse a una ciencia naturalista y tecnocientífica, devaluando la fundamentación epistemológica en nombre de 
la eficacia (Conti, 2008; Pellegrino, 1979). Desde esta perspectiva, se polarizan los hechos observables y su sentido, priorizando los primero sobre los segundos. No obstante, ninguna ciencia puede ser concebida como puro datos y además pretender ser una consideración puramente teórica de esos datos (Rovaletti, 1994). Todo enunciado sobre el hombre, incluso el puramente científico-objetivo encierra ya un conjunto de presupuestos antropológicos y metafísicos (Landgrebe, 1969).

Ahora bien, la imposibilidad de las ciencias naturalistas contemporáneas, que exilian al hombre de su mundo y limitan a un ser teleológico a la mera sumatoria de reflejos, de dar cuenta de la la afectividad y la corporalidad humana, devela la necesidad de una nueva psicología fundamentada ontológicamente en el ser del hombre (Straus, 1966/1971). Paragonando a Heidegger (1927/2012), esto implica una revisión de los conceptos fundamentales que determinan la comprensión previa de la región que sirve de base a todos los objetos temáticos de una ciencia y que orientan por ello toda búsqueda científica.

Precisamente, para Straus, es necesario fundar "una psicología que no sea la psicología de un aparato, 'no una psicología fisiológica sino psicológica'. Si puede decírselo así, una psicología que estudie al hombre como ser capaz de experiencia en su relación yo-mundo." (Straus, 1966/1971, p. 279). Por ello, hacia el final de su vida, Straus propone una nueva concepción del ser humano, desarrollada a partir de su experiencia clínica e investigaciones fenomenológicas sobre la condición humana:

En vez de continuar torturándonos en el vano esfuerzo de definir la conciencia, vamos a definir como un organismo experimentante al capaz, en su propia motilidad como sujeto corporal, de captar el Mundo como Mundo; vamos a definir como conciencia a un humano que es capaz de dirigirse al todo del que él mismo es una parte (Straus, 1969, p.76-77).

Ahora, se propone como objeto de estudio de la ciencia psicológica un ser bio-psico-social que no es ni enteramente fuera de sí como la res extensa, ni enteramente en sí como el espíritu puro. Se trata de una subjetividad encarnada, que se siente a sí misma cuando siente otra cosa, y su acción propia está expuesta sin cesar a otras influencias, por ello su intrínseca vulnerabilidad (Straus, 1935/1963). Desde la presente perspectiva, todo análisis del comportamiento del sujeto, desde el mero suspiro hasta la composición de una obra de arte, deben ser analizado en su significación existencial, es decir, como variaciones de la relación yo-mundo, como modos de estructurar la propia presencia frente a la totalidad de la que el sujeto forma parte. Así, la rigidez de las cenas protocolares, el esfuerzo del alumno por evitar bostezar o el liberarse de las restricciones en la danza improvisada, constituyen modos diversos de posicionarse frente a una situación, los otros y frente a sí-mismo. Esta nueva concepción del objeto de estudio de la Psicología permite establecer un nuevo marco, tanto para las futuras investigaciones experimentales sobre la corporalidad y la afectividad como para la comprensión de la experiencia del hombre alienado.

Por una parte, en el campo de la neuropsicología, Varela (1995) advierte la necesidad de un cambio epistemológico radical. Frente al desencanto de lo abstracto en la psicología, esa tradición racionalista, que se mueve en la atmósfera de lo general, lo lógico y lo bien definido, se postula el retorno a la subjetividad encarnada inscripta en una situación biográfica única e irrepetible. Propone como tarea de la neuropsicología, ya no el descubrir los efectos de un mundo independiente sobre un aparato intracraneal, sino el indagar el modo en el que el sistema nervioso conecta las esferas sensoriales y motora en el individuo concreto (Varela, 1995). Precisamente, la perspectiva strausiana sobre la afectividad y el movimiento teleológico, la cual quiebra los tres dualismos que recorren las investigaciones de la psicología objetivista sobre la corporalidad y la afectividad -sujeto-objeto, receptividad-actividad e interior-exterior-, se ofrece como un marco filosófico fructífero para el cambio de paradigma exigido por Varela.

En segundo lugar, la concepción antropológica de Straus introduce al hombre alienado como sujeto y no como objeto. Desde la psicología naturalista la alienación es acotada a una disfunción biológica, reduciéndola así a un manojo de escombros y un agregado anárquico de síntomas (Straus, 1966/1971; 1967b). Por el contrario, para Straus, las psico(pato)patologías expresan diversos modos de relaciones yo-mundo, plenamente significativos, en los que priman la imposibilidad de la existencia de decidir sobre el curso de su biografía (1967b). La alineación constituye un cambio radical en la situación primaria [Ur-situation] del hombre : incapaz de mantener su o-posición frente al mundo, el individuo pierde la soberanía sobre su existencia y los márgenes de libertad necesarios para poder comportarse de una forma $\mathrm{u}$ otra se ven restringidos (Straus, 1966/1971). El poder comportar-se no se refiere a un comportamiento ajustado a una norma sino a la autonomía inherente al hombre que decide sobre su destino (Blankenburg, 1983). Desde esta perspectiva, es posible erigir una terapia que esté al servicio del alienado, del semejante cuya autonomía se ve restringida por la opresión de las circunstancias, y no de la enfermedad en tanto categoría abstracta.

A modo de conclusión, es necesario destacar que la presente fundamentación strausiana de la Psicología no resulta de un interés exclusivamente epistemológico, sino que implica una perspectiva ética. Para el autor, la reducción la existencia a un mero fenómeno biológico pone a las ciencias del hombre ante una encrucijada moral en la medida en que arriesgan a quitarle al individuo su dignidad y libertad. Por el contrario, 
la antropología filosófica strausiana devela la diferencia ontológica entre los meros objetos y el ser humano. Este último se caracteriza por el establecimiento de relaciones significativas con el mundo, primariamente, mediante su comportamiento teleológico y la experiencia sensorial (Straus, 1941; 1969).

\section{Referencias}

Barbaras, R. (2004) Affectivity and movement: The sense of sensing in Erwin Straus. Phenomenology and the cognitive sciences, 3 (1): 215-228. URL: https://link.springer.com/article/10.1023/ B:PHEN.0000040834.95059.0a

Binswanger, L. (1995/2006). Obras Escogidas (1995/2006). España: RBA.

Binswanger, L. (1957/2012). O caso Suzanne Urban. Psicopatologia Fenomenológica Contemporânea, 1 (1): 198-344. URL: http://www.revistapfc.com.br/rPFCwordpress/wp-content/ uploads/2017/01/198_BinswangerL_O-caso-Suzanne-Urban.pdf

Blankenburg, W. (1983). La psicopatología como ciencia básica de la psiquiatría. Revista chilena de Neuropsiquiatría, 21(1), 177-188.

Chessick, R. D. (1999). The phenomenology of Erwin Straus and the epistemology of psychoanalysis. American Journal of Psychotherapy 53(1): 82-95.

Conti, N. (2008). Filosofía y psiquiatría. Sinopsis, 40: 17-22.

Eng, E. (1976). Locating Erwin Straus. Journal of Phenomenological Psychology, 7(1), 1-14. URL: https://search.proquest.com/ openview/8a5a77ed9bbf239ce0d48435b4216de4/1?pq-origsite $=$ gscholar\&cbl $=1821473$

Fischer, W. (1976). Erwin Straus and the phenomenological approach to psychopathology. Journal of Phenomenological Psychology, 7(1), 95-115. URL: https://search.proquest. com/openview/1433834b6707fd098aaaa1c7fd411531/1?pq-origsite =gscholar \&c$\underline{\mathrm{bl}=1821473}$

Galimberti, U. (1987). Psichiatria e Fenomenologia. Milano: Feltrinelli.

Gennart, M. (1986). Une phénoménologie des données hylétiques est-elle possible?: A propos de Vom Sinn der Sinne de Erwin Straus. Études phénoménologiques, 2(4), 19-46.

Heidegger, M. (2012). Ser y Tiempo. Madrid: Editorial Trota
Husserl, E. (1913/1962). Ideas relativas a una fenomenología pura y una filosofía fenomenológica. México: Fondo de Cultura Económica.

Husserl, E. (1952/1997). Ideas II. Investigaciones fenomenológicas sobre la constitución. Mexico: Universidad Nacional Autónoma de México.

Husserl, E. (1954/2008). La crisis de las ciencias europeas y la fenomenología trascendental. Buenos Aires: Prometeo Libros.

Khun, R. (1996). El síntoma como hecho a ser comprendido; En torno a la frase 'en la psiquiatría los síntomas son ellos mismos hechos a ser comprendidos'. En M. L. Rovaletti (Ed.) (1996). La problemática del cuerpo en el pensamiento actual (pp. 325-334).

Landgrebe, L. (1963/1968). El camino de la fenomenología. El problema de una experiencia originaria. Buenos Aires: Editorial Sudamericana.

Landgrebe, L. (1957/1969). La filosofía actual. Caracas: Monte Avila Editores.

Landgrebe, L. (1968/1975). Fenomenología y Historia. Caracas: Monte Avila Editores

Landgrebe, L. (1977/2017). El problema de la teleología y la corporalidad en la fenomenología y en el marxismo. Acta Mexicana de Fenomenología. Revista de investigación Filosófica y Científica, 10(2): 93-124.

Lindeboom, G. A. (1978). Descartes and Medicine. Amsterdam: Rodopi NV.

MacIntyre A. (1981). After Virtue. Notre Dame: University of Notre Dame Press.

Maldiney, H. (1973). Le devoilement de la dimension esthetique dans la phenomenologie d'Erwin Straus. En Regard Parole Espace, (pp 124146) Lausanne: L'Age d'Homme.

Marleu-Ponty, M. (1945/1993). Fenomenología de la percepción. México-Argentina: Fondo de Cultura Económica.

Moran, D. (2012). Husserl's crisis of the European sciences and transcendental phenomenology: An introduction. Cambridge: Cambridge University Press.

Moss, D. (1981). Erwin straus and the problem of individuality. Human Studies, 4, 49-65. URL: https://philpapers.org/rec/MCKESA

Pellegrino, E.D. (1979). Humanism and the Physician. Knoxville: University of Tennessee Press. 
Ramos Gorostiza, P. \& Gonzalez Calvo, J. M. (1995). El sentido de la Psicopatología y el papel de la fenomenología. Contribución a una controversia. Actas Luso-españolas de Neurología y Psiquiatría, 23 (5), 281-286.

Rovaletti, M. L. (1994). La Psicología fenomenológica y el contexto teórico de su constitución. En Rovaletti, M. L. (ed). Psicología y Psiquiatría Fenomenológica (pp. 1-22). Bs. As: Cátedra de Psicología Fenomenológica y Existencial.

Rovaletti, M. L. (2016). ¿Cómo pensar una clínica fenomenológica? Vertex Revista Argentina de Psiquiatría, vol. XXVII, 47-55.

San Martín, J. (1986). La estructura del método fenomenológico. Madrid: Universidad Nacional de Educación a Distancia.

Spiegelberg, H. (1972). Phenomenology in Psychology and Psychiatry: A Historical Introduction. Evanston: Northwestern University Press.

Straus, E. (1936/2015). El movimiento vivido. Limite 10 (33): 3-15. URL: http://www.redalyc.org/ $\underline{\mathrm{html} / 836 / 83642663001 /}$

Straus, E. (1941). Education in a Time of Crisis', Black Mountain College Bulletin, No.7.

Straus, E. (1948). On obsession. A clinical and methodological study. New York: Coolidge Foundation.

Straus, E. (1952). The upright posture. En Natason, M. (Ed). Essays in Phenomenology (pp. 164- 192). Netherlands: Springer.

Straus, E. (1935/1963). The Primary World of Senses. A Vindication of Sensory Experience. London: The Free Press of Glencoe, Collier-Macmillan Limited.

Straus, E. (1965a). Born to see, bound to behold. Tijdschrift Voor Filosofie, 27 (4): 659- 688. URL: http:// www.jstor.org/stable/40881226

Straus, E. (1965b). The sense of the senses. The Southern Journal of Philosophy, 3(4), 192201. URL: http://onlinelibrary.wiley.com/ doi/10.1111/j.2041-6962.1965.tb01710.x/full

Straus, E. (1966/1971). Psicología fenomenológica. Buenos Aires: Editorial Paidos.

Straus, E. (1967a). An existential approach to time. Annals of the New York Academy of Sciences, 138(2), 759-766.

Straus, E. (1958/1967b). Estesiología y alucinaciones. En May, R. Angel, E., Ellenberger, H. (Eds.) (1958/1967). Existencia. Nueva dimensión en Psiquiatría y Psicología (pp. 177 - 211). Madrid: Gredos.
Straus, E. (1969). Psychiatry and philosophy. En Straus, E., Natanson, M., \& Ey, H. (Eds.) Psychiatry and philosophy (pp 1-84). New York: Springer.

Straus, E. (1975): The Monads Have Windows. En Phenomenological Perspectives: Historical and Systematic Essays in Honor of Herbert Spiegelberg (p. 130-150). Netherlands: Springer.

Thinés, G. (1977/1978). Fenomenología y Ciencias de la Conducta. Madrid: Pirámides.

Titleman, P. (1976) A Phenomenological Approach to Psychopathology: The Conception of Erwin Straus. Journal of Phenomenological Psychology, 7(1): 13-33. URL: https://search. proquest.com/openview/8a5a77ed9bbf239c4c3d77379a728003/1?pq-origsite $=$ gscholar\&c$\underline{\mathrm{bl}=1821473}$

Varela, F. J. (1995). The re-enchantment of the concrete. Some biological ingredients for a nouvelle cognitive science. En Steels, L., \& Brooks, R. (eds), The artificial life route to artificial intelligence: building embodied, situated agents (pp. 11-20). Estado Unidos: Lawrence Erlbaum

Waldenfels, B. (2008). Lassie corporelle des sentimentrs. En Escoubas, E, Tengelyi, L. (Dir.). Affect et affectivité dans la philosophie moderne et la phénoménologie (pp. 201 - 220). Paris: L’Harmat$\tan$.

Zaner, R.M. (1988). Ethics and the Clinical Encounter. Englewood Cliffs, N.J.: Prentice-Hall, Inc.

Zaner, R.M. (2004) The discipline of the "norm": a critical appreciation of Erwin Straus. Human Studies, 27: 37-50. url: https://link.springer.com/article/10.1023/B:HUMA.0000012248.54939.42

Zirión, A. (2009). El resplandor de la afectividad. Acta Fenomenológica Latinoamericana, 3, 139-153.

Sebastián Eduardo Mendl (https://orcid.org/0000-00031023-4719), Psicólogo, Instituto de investigaciones Facultad de Psicología, Universidad de Buenos Aires. Proyecto UBACyT, código 20020130100823BA, dirigido por Prof. emérita Dra. Lucrecia Rovaletti. E-mail: sebastianemendl@gmail.com

Recebido em 05.02.2018 Decisão Editorial em 08.02.19 Aceito em 03.04.19 